\title{
EARING IN DEEP-DRAWING STEELS
}

\author{
G. J. DAVIES, D. J. GOODWILL and J. S. KALLEND \\ Department of Metallurgy and Materials Science, University of Cambridge \\ Pembroke Street, Cambridge, U.K.
}

(Received March 20, 1972)

\begin{abstract}
The variations of the fourth-order coefficients of the crystallite orientation distribution function, with rolling reduction have been determined after cold-rolling and annealing for a deep-drawing quality rimming steel and an aluminium-killed steel. These coefficients influence drawability and earing behaviour and by the manipulation of the coefficients in the distribution function of a $60 \%$ cold-rolled and annealed rimming steel, a hypothetical nonearing sheet texture has been derived. By comparison with the actual sheet texture those textural components which most affect earing behaviour are identified.
\end{abstract}

\section{INTRODUCTION}

The effects of normal and planar anisotropy of mechanical properties on the deep-drawing performance of sheet metals have been variously reported. ${ }^{1-5}$ In particular, the relationship between anisotropy and the appearance of "earing" has attracted considerable attention. The earing phenomenon has been reviewed by Wright $^{6}$ and by Grewen. ${ }^{7}$ Although earing in sheet metal is known to be determined by mechanical anisotropy attributable to the existence of crystallographic preferred orientation (texture) in the sheet, there are few quantitative treatments available. ${ }^{5,8,9}$ One of the most important aspects of texture study is the development of methods for changing the texture towards the ideal. For control of earing this requires clear identification of the textural components contributing most strongly to the mechanical anisotropy. Although empirical methods have been developed with some success, ${ }^{5,7}$ fundamental analyses are still needed.

The application of spherical harmonic analysis methods in texture research ${ }^{10-14}$ has yielded quantitative descriptions of texture, in the form of crystallite orientation distribution functions, which can be used to predict elastic and plastic anisotropy with some accuracy. The object of the present paper is to show how the crystallite orientation distribution function can be used to study earing in sheet steels of deep-drawing quality. The procedures described are of more general applicability.

\section{ANALYTICAL BASIS}

Pursey and $\operatorname{Cox}^{15}$ in their analysis of elastic anisotropy in cubic crystals showed that only four independent coefficients of the crystallite orientation distribution function enter into the expression for the polycrystalline elastic constants. The usual expression for the distribution function, ${ }^{10}$ is

$$
\begin{aligned}
w(\psi, \xi, \phi)= & \sum_{l=0}^{\infty} \sum_{m=-l}^{l} \sum_{n=-}^{l} W_{l m n} Z_{l m n}(\xi) \exp (-i m \psi) \\
& \exp (-i n \phi)
\end{aligned}
$$

where $\psi, \theta\left(=\cos ^{-1} \xi\right)$ and $\phi$ are the Euler angles describing the crystallite orientation, $W_{l m n}$ are the coefficients of the generalised spherical harmonic series and $Z_{l m n}(\xi)$ is a generalisation of the associated Legendre function (the so-called augmented Jacobi polynominals ${ }^{12}$ ). In this function it is the independent coefficients, $W_{000}, W_{400}, W_{420}$ and $W_{440}\left(W_{4 m 4}\right.$ being linearly dependent on $\left.W_{4 m 0}\right)$ which determine elastic anisotropy.

Similarly, it has been shown ${ }^{16}$ that these fourth order coefficients largely determine the plastic anisotropy of cubic metals. This is a consequence of the observation that when the anisotropy of plasticity of a cubic single crystal deforming by polyslip according to the Bishop and Hill theory ${ }^{17,18}$ is expressed as a series of generalised spherical harmonics the coefficients, $G_{l m n}(q)$ of higher than fourth order are an order of magnitude 
smaller than those for the zeroth $\uparrow$ or fourth order (see Table I).

\section{TABLE I}

Mean absolute values of the coefficients $G_{l m n}(r)$ for various orders of the expansion of the function describing flow stress anisotropy according to the Bishop and Hill theory, with contraction ratio $r$

\begin{tabular}{cl}
\hline Order & Mean absolute value of $G_{l m n}(r)$ \\
\hline 0 & 4.470 \\
2 & 0 \\
4 & 0.191 \\
6 & 0.012 \\
8 & 0.022 \\
10 & 0.003 \\
12 & 0.005 \\
14 & 0.003 \\
\hline
\end{tabular}

In making the analysis of plastic anisotropy ${ }^{16}$ the series coefficients, $G_{l m n}(q)$ are evaluated for successively different values of an assumed contraction ratio, $q$, given by

$$
q=\frac{d \epsilon_{y y}}{d \epsilon_{y y}+d \epsilon_{z z}}=\frac{r}{1+r}
$$

where $d \epsilon_{y y}$ and $d \epsilon_{z z}$ are strains in the width and thickness directions, respectively, and $r$ is the conventional strain ratio (width strain/thickness strain).

In deriving Table I the values of $G_{l m n}(q)$ have been calculated for contraction ratios $q=0,0.1$, $0.2 \ldots 1.0$ for different orders of $l$ up to and including the fourteenth. In predicting the plastic anisotropy of polycrystals ${ }^{14,16}$ this information is combined with the crystallite orientation distribution data. If the lower-order texture coefficients, $W_{l m n}$, are of the same order of magnitude (this is normally the case), the contribution of product terms of order greater than four can be neglected. Cross terms are not present because of the orthogonal properties of generalised spherical harmonic functions.

The analysis of drawability can be undertaken in a parallel way. Hosford and Backofen ${ }^{2}$ showed that good drawability in sheets of cubic metals is obtained when the parameter, $\beta$, given by the ratio

†The zeroth order coefficients give rise to orientation independent properties. of the yield strengths under the plane-strain conditions existing in the cup wall and flange respectively, is large. $\ddagger$ This parameter can easily be related to the crystallographic texture. $^{2}$ Kallend $^{19}$ showed that for the coefficients of the crystallite orientation distribution function a large negative $W_{400}$ gives a large $\beta$ value and thus good drawability. Furthermore, a positive $W_{420}$ controls the tendency to form two ears at $90^{\circ}$ and $270^{\circ}$ from the rolling direction and a positive $W_{440}$ leads to four ears at $0^{\circ}, 90^{\circ}, 180^{\circ}$ and $270^{\circ}$ to the rolling direction. Correspondingly, a negative $W_{420}$ gives two ears at $0^{\circ}$ and $180^{\circ}$ and a negative $W_{440}$ gives four ears at $45^{\circ}, 135^{\circ}, 225^{\circ}$ and $315^{\circ}$ to the rolling direction. The earing tendency is decreased as the values of $W_{420}$ and $W_{440}$ tend to zero.

The present work involves firstly the manipulation of the fourth-order coefficients of the crystallite orientation distribution function obtained experimentally for a commercial rimming steel and the correlation of changes in the drawability and earing tendency with changes in the textural components of the distribution. Subsequently the results are applied to a commercial killed steel. The initial texture analysis was made using rimming steel because the orientation distribution function for this steel was such as to allow the ready identification of the different texture components. The crystallite orientation distribution function for the killed steel obscured small changes in intensity due to its higher overall severity.

\section{EXPERIMENTAL}

The steels considered were commercial deepdrawing quality rimming steel and killed steel whose compositions are given in Table II. Samples of the steels were cold rolled to different reductions using an oil lubricant. Rolling was followed by a simulated commercial annealing treatment, viz.: slow heat $\left(25^{\circ} \mathrm{C}\right.$ per hour) to $700^{\circ} \mathrm{C}$, hold for 20 hours, slow cool to room temperature $\left(25^{\circ} \mathrm{C}\right.$ per hour), in an atmosphere of argon at reduced pressure. Texture measurements were made on samples prepared by the composite specimen method of Elias and Heckler ${ }^{20}$ using a Siemens texture goniometer with filtered MoK $\alpha$ radiation. $\{110\},\{200\}$

\footnotetext{
IIt should be noted that the $\beta$-parameter is difficult to measure experimentally. For this reason the plastic strain ratio, $r$, is more frequently used as a measure of drawability.
} 


\section{TABLE II}

Composition and hot process treatment of rimming and killed steels

\begin{tabular}{lcc}
\hline Element & Rimming steel wt. \% & Killed steel wt. \% \\
\hline $\mathrm{C}$ & 0.020 & 0.035 \\
$\mathrm{~S}$ & 0.022 & 0.022 \\
$\mathrm{P}$ & 0.006 & 0.008 \\
$\mathrm{Mn}$ & 0.27 & 0.24 \\
$\mathrm{Cu}$ & 0.090 & 0.030 \\
$\mathrm{Ni}$ & 0.060 & 0.025 \\
$\mathrm{Sn}$ & 0.020 & 0.010 \\
$\mathrm{Si}$ & 0.01 & - \\
$\mathrm{Cr}$ & 0.070 & 0.0074 \\
$\mathrm{~N}$ & 0.0028 & 0.047 \\
Total Al & 0.030 & 0.040 \\
Sol Al & 0.01 & $882-904^{\circ} \mathrm{C}$ \\
Finishing & & \\
Temperature & $898-937^{\circ} \mathrm{C}$ & (mean $893^{\circ} \mathrm{C}$ ) \\
Coiling & $\left(\right.$ mean $920^{\circ} \mathrm{C}$ ) & \\
Temperature & $653-620^{\circ} \mathrm{C}$ & \\
The section used for experimentation was from the centre \\
of the coil.
\end{tabular}

TABLE III

Euler angles for given ideal orientations

\begin{tabular}{|c|c|c|c|c|}
\hline$h k l$ & $u v w$ & $\theta$ & $\phi$ & $\psi$ \\
\hline 100 & 011 & $\begin{array}{r}90 \\
90 \\
0\end{array}$ & $\begin{array}{c}45 \\
45 \\
(\phi-1\end{array}$ & $\begin{array}{r}0 \\
90 \\
=45\end{array}$ \\
\hline 110 & 001 & $\begin{array}{l}45 \\
45 \\
90\end{array}$ & $\begin{array}{r}0 \\
90 \\
45\end{array}$ & $\begin{array}{r}90 \\
90 \\
0\end{array}$ \\
\hline 320 & 001 & $\begin{array}{l}56.3 \\
90 \\
33.7 \\
90\end{array}$ & $\begin{array}{c}0 \\
33.7 \\
0 \\
56.3\end{array}$ & $\begin{array}{r}90 \\
0 \\
90 \\
0\end{array}$ \\
\hline 100 & 001 & $\begin{array}{r}0 \\
90 \\
90 \\
90 \\
90\end{array}$ & $\begin{array}{c}\phi+ \\
0 \\
0 \\
90 \\
90\end{array}$ & $\begin{array}{c}0,90 \\
0 \\
90 \\
0 \\
90\end{array}$ \\
\hline 111 & $u v w$ & 54.7 & 45 & any \\
\hline 110 & $u v w$ & $\begin{array}{l}45 \\
45 \\
90\end{array}$ & $\begin{array}{r}0 \\
90 \\
45\end{array}$ & $\begin{array}{l}\text { any } \\
\text { any } \\
\text { any }\end{array}$ \\
\hline 100 & $u v w$ & $\begin{array}{r}0 \\
90 \\
90\end{array}$ & $\begin{array}{l}(\phi+ \\
0 \\
90\end{array}$ & $\begin{array}{r}=\text { any } \\
\text { any } \\
\text { any }\end{array}$ \\
\hline
\end{tabular}

and $\{211\}$ pole figures were used to determine the coefficients of the crystallite orientation distribution functions for each pre-annealing reduction.

Constant $\phi$ sections of the crystallite orientation distribution function were plotted automatically using a curve plotter controlled by a contouring program. Specific orientations were indexed by reference to the charts published previously. ${ }^{21}$ Table III lists orientations of interest in terms of the corresponding Euler angles $\theta, \phi$ and $\psi$.

Using the experimental distribution function for a sample of rimming steel given a treatment close to that adopted commercially (viz. $60 \%$ cold reduction prior to annealing) the effect of setting $W_{420}$ and $W_{440}$ to zero both separately and together was evaluated. In each case constant- $\phi$ sections were reconstituted and comparison made with the original plots. In this way those textural components influencing earing could be identified.

The crystallite orientation distribution function data were also used to predict the planar anisotropy of the yield stress and of the plastic strain ratio using the method of Bunge and Roberts ${ }^{11}$ and Kallend and Davies. ${ }^{14,22}$

\section{RESULTS AND DISCUSSION}

\section{Fourth Order Texture Coefficients}

Figure 1 shows the variation in the $W_{4 m 0}$ coefficient with increasing true strain from cold rolling prior to annealing for the rimming steel. As mentioned above, a large negative $W_{400}$ gives rise to a large $\beta$-value and good drawability. This is achieved by increasing the rolling reduction prior to annealing although the rate of change decreases significantly above reductions of about $60 \%$.

$W_{420}$ is always negative and nearly independent of reduction. Thus a pair of ears at $0^{\circ}$ and $180^{\circ}$ are expected after all reductions. $W_{440}$, however, is negative below $30 \%$ reduction and above $88 \%$ reduction. The positive values reach a maximum at $60 \%$ reduction. Thus this coefficient should give rise to ears at $45^{\circ}, 135^{\circ}, 225^{\circ}$ and $315^{\circ}$ outside the range $30-88 \%$ reduction and at $0^{\circ}, 90^{\circ}, 180^{\circ}$ and $270^{\circ}$ within this range. Experimental observations ${ }^{4}$ confirm these predictions. This is also confirmed by results presented in Figure $2 \dagger$ which shows the variation in ear height with rolling reduction for a

†Data supplied by R. C. Hudd, British Steel Corporation, Strip Mills Division. 


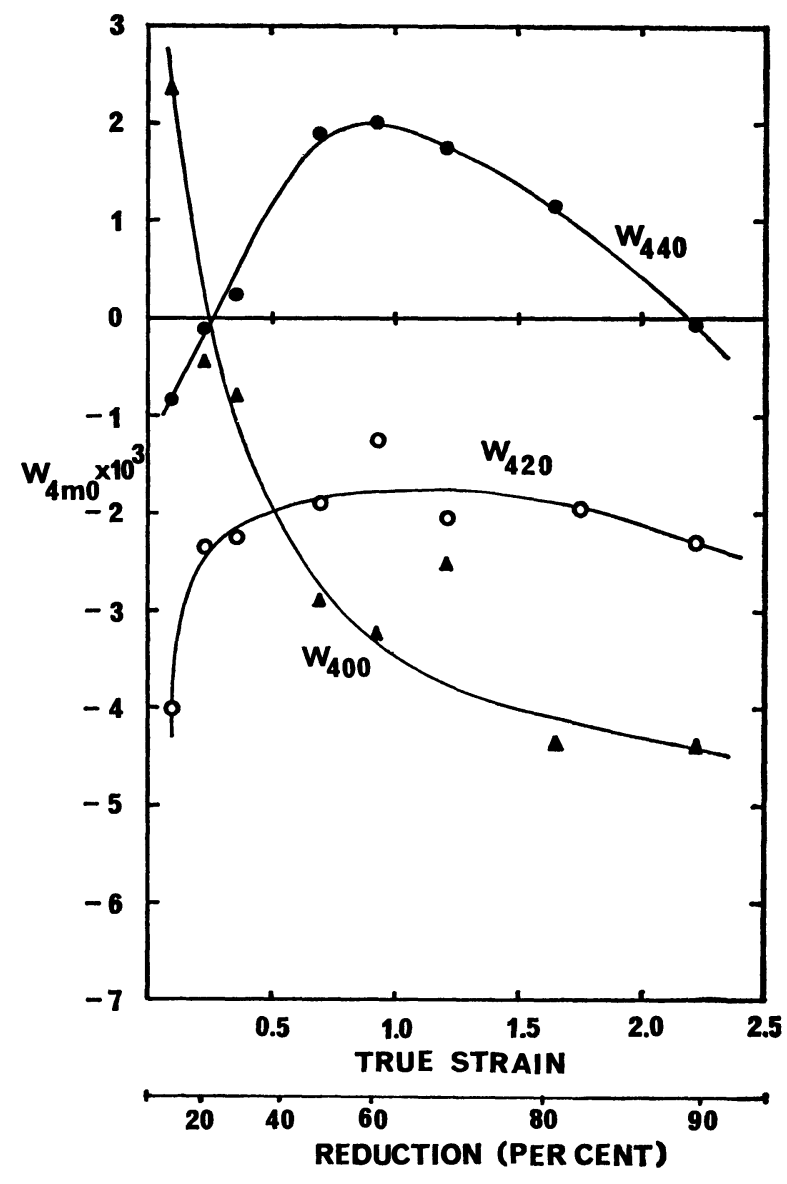

FIGURE 1 The variation of the $W_{4 m 0}$ texture coefficients with rolling reduction prior to annealing for the rimmed steel.

rimming steel similar to the one used to derive Figure 1.

The best earing behaviour is obtained by minimising the deviation of the top of the cup from being flat. In some cases this can be ac'uieved by using balanced textures. The procedures developed empirically ${ }^{7,24}$ for cubic metals to minimise earing by combining $0^{\circ} / 90^{\circ}$ and $45^{\circ}$ earing, effectively minimise $W_{440}$. In the present case since $W_{420}$ is independent of reduction optimisation would involve minimising $W_{440}$ to as large a degree as possible consistent with increasing drawability. Notwithstanding this, it should be noted that the $W_{420}$ and $W_{440}$ coefficients are not crystallographically independent since textural components contributing to $W_{420}$ can also contribute to $W_{440}$.

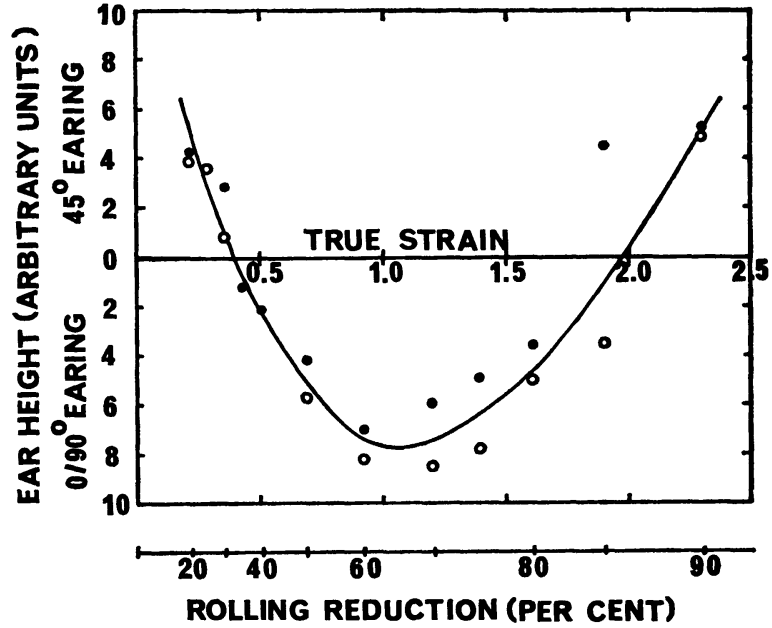

FIGURE 2 The variation in ear height with rolling reduction for a rimming steel similar to the one used to derive Fig. 1.

Sample 1 from the front end of the coil.

Sample 2 from the back of the coil.

In commercial practice the cold reduction adopted depends on the final thickness and properties required of the sheet. Figure 1 shows that $W_{400}$ does not change much beyond $60 \%$ reduction. For good drawability and in applications where earing is not disadvantageous, reductions of about $60 \%$ are commonly employed. Where non-earing properties are particularly desirable, the cold reduction will be closer to either 30 or $88 \%$ and this may result in some loss of drawability. ${ }^{24}$

In Figures 3, 4 and 5 only the series coefficients $W_{000}$ and $W_{400}, W_{000}$ and $W_{420}$, and $W_{000}$ and $W_{440}$ together with the linearly dependent coefficients have been used, respectively. $W_{000}$, the texture independent coefficient, effectively sets the contours for random texture to be everywhere unity. Examination of these figures with reference to Table III and the indexing charts ${ }^{21}$ shows that $W_{400}$ is controlled mainly by the amount of orientations with $\{111\}$-planes parallel to the plane of the sheet. This was expected, since it is well known that a large proportion of $\{111\}$-oriented planes in a textured material gives good drawing properties, ${ }^{1}$ and, as stated above, the overall level of any parameter used to quantify drawability is dependent on $W_{400}$.

From Figure 4 it can be seen that the orientations which most affect $W_{420}$ are those close to $\{110\}$ 

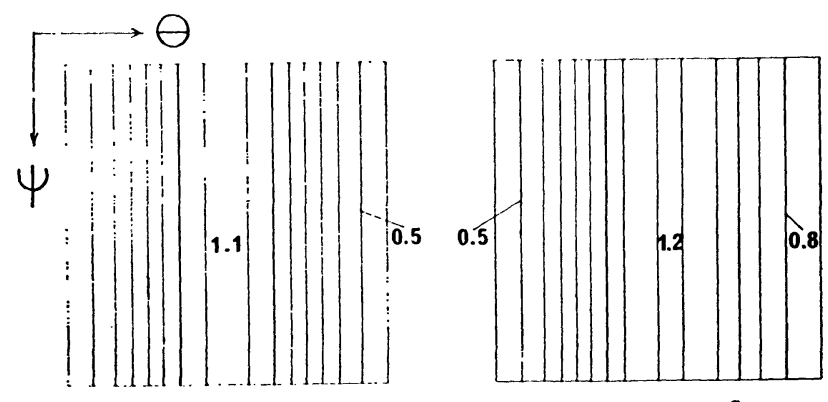

$\phi=0^{\circ}$

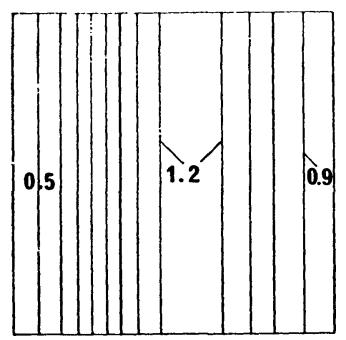

$\phi=25^{\circ}$

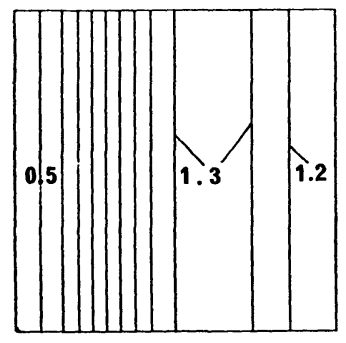

$\phi=45^{\circ}$ $\phi=20^{\circ}$

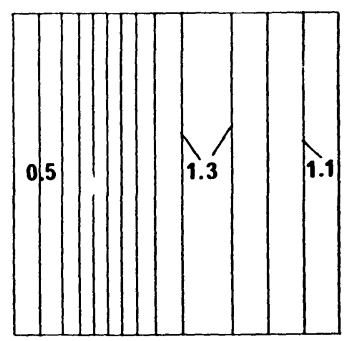

$\phi=35^{\circ}$

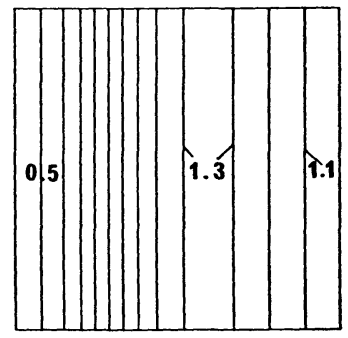

$\phi=55^{\circ}$

FIGURE 3 The crystallite orientation distribution function for the $W_{000}$ and $W_{400}$ coefficients alone, for rimmed steel rolled $60 \%$ and annealed.

$\langle 001\rangle$. These orientations, if present, will cause the "two ear" phenomenon. Figure 5 shows that the major orientations contributing to the "four ear" phenomenon are those with $\langle 001\rangle$ parallel to the rolling direction, in particular the cube texture, $\{100\}\langle 010\rangle$. Since these include $\{110\}$ $\langle 001\rangle$, and cube texture is not present to any great extent in the steel, it is clear that the amount of orientations close to $\{110\}\langle 001\rangle$ is going to control the extent to which earing occurs on deep drawing in rimming steel.

The levels of contours for the peak orientations in Figure 3 should be made as large as possible for $W_{400}$ to be large and if good drawability is to be achieved. This will be a result of normal anisotropy
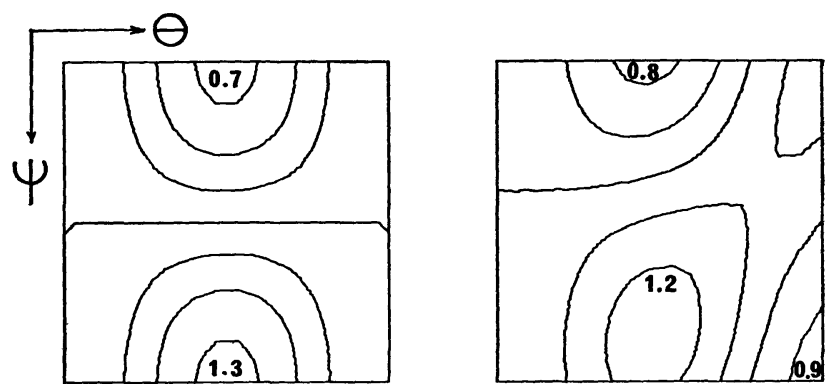

$\phi=20^{\circ}$
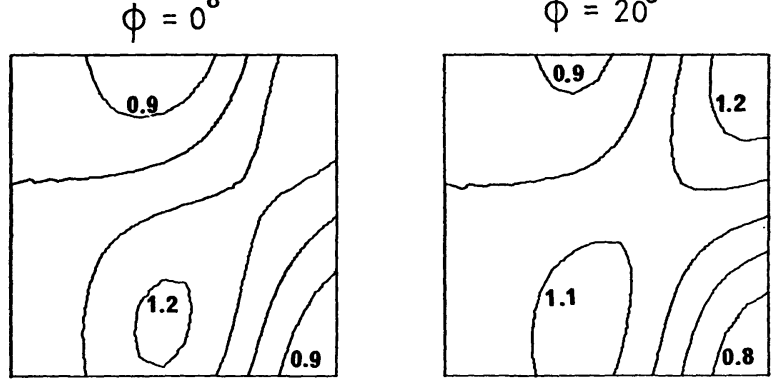

$\phi=25^{\circ}$

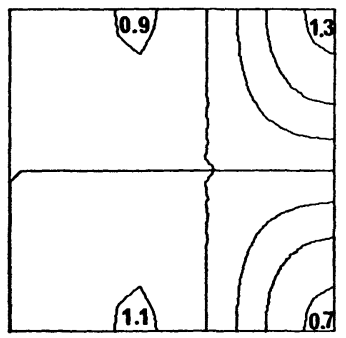

$\phi=45^{\circ}$

$\phi=35^{\circ}$

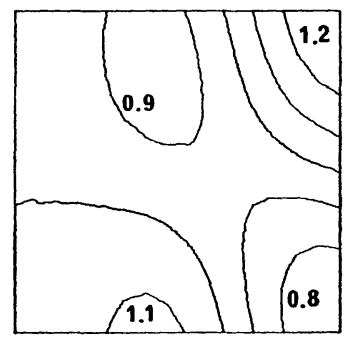

$\phi=55^{\circ}$

FIGURE 4 The crystallite orientation distribution function for the $W_{000}$ and $W_{420}$ coefficients alone, for rimmed steel rolled $60 \%$ and annealed.

and hence will involve the preferred alignment of plane normals. \{111\}-planes are most conducive to favourable drawability. $\{110\}$-planes give good drawability while $\{100\}$-planes will give the minimum drawing performance. From Figures 4 and 5 it can be seen that $W_{420}$ and $W_{440}$ should be minimised as far as possible to avoid earing. This involves planar anisotropy the extent of which will be determined by the degree of alignment of crystallographic directions. Orientations with $\langle 100\rangle$ in the rolling direction should be eliminated as far as possible. In addition the amount of the $\{100\}$ $\langle 011\rangle$ component should be increased in order to reduce $W_{440}$ and get a reduction in ear height, but this would decrease the value of $W_{400}$. 


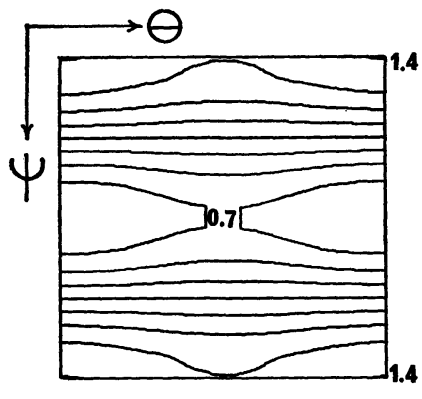

$\phi=0^{\circ}$

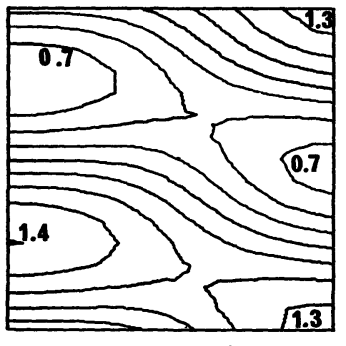

$\phi=25^{\circ}$
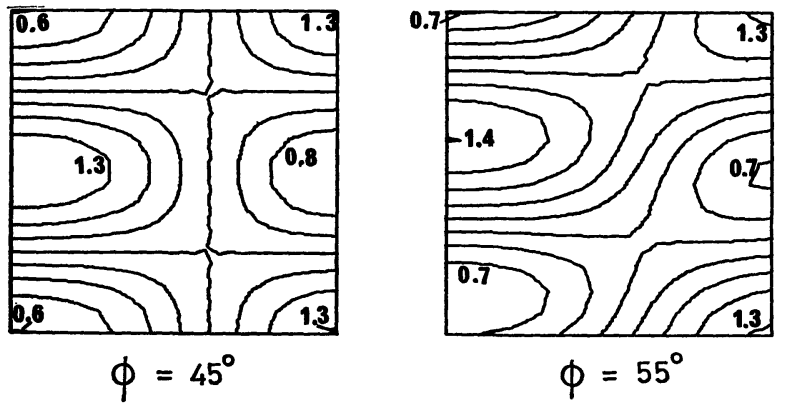

$\phi=55^{\circ}$

FIGURE 5 The crystallite orientation distribution function for the $W_{000}$ and $W_{440}$ coefficients alone, for rimmed steel rolled $60 \%$ and annealed.

Variations in the Cold-Rolled Texture of Rimming Steel Produced by Manipulation of the Texture Coefficients

Constant- $\phi$ sections of the crystallite orientation distribution function for the sample of rimmed steel cold rolled $60 \%$ and annealed involving all coefficients up to twentieth order are presented in Figure 6. Setting $W_{420}$ to zero produces the new set of constant $\phi$-sections given in Figure 7. Likewise, $W_{440}$ is set to zero in Figure 8, and both $W_{420}$ and $W_{440}$ are zero in Figure 9.

Removing $W_{420}$ measurably affects orientations close to $\{110\}\langle 001\rangle$ (including $\{320\}\langle 001\rangle$ ), as does the removal of $W_{440}$. This latter coefficient affects all orientations of the form $\{h k l\}\langle 001\rangle$.

Setting both $W_{420}$ and $W_{440}$ to zero has an even more marked effect. Figure 9 represents the hypo-

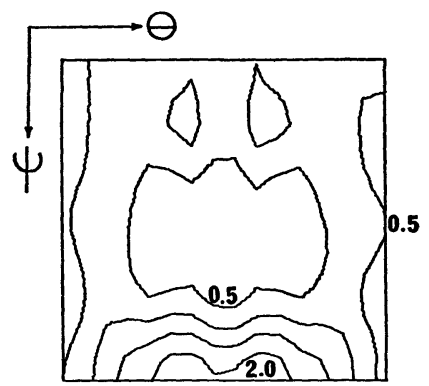

$\phi=0^{\circ}$

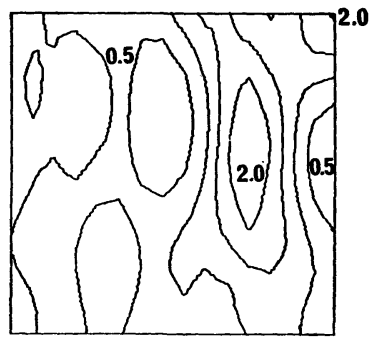

$\phi=25^{\circ}$

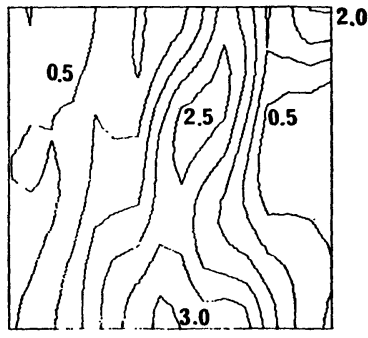

$\phi=45^{\circ}$

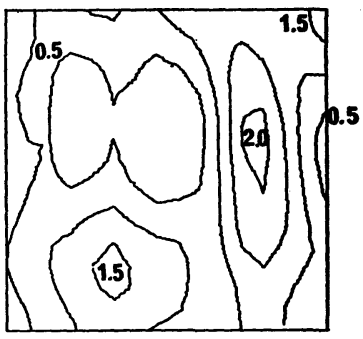

$\phi=20^{\circ}$

25

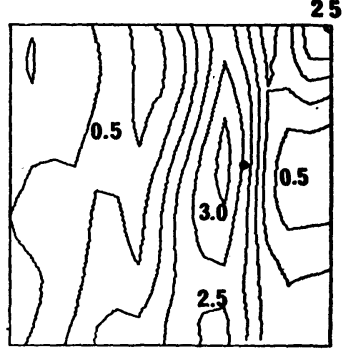

$\phi=35^{\circ}$

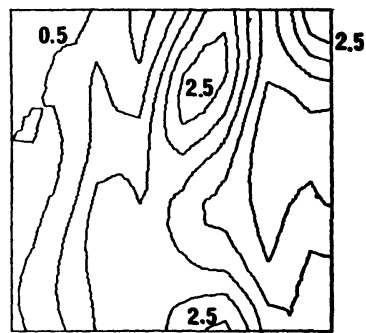

$\phi=55^{\circ}$

FIGURE 6 The crystallite orientation distribution function for rimmed steel cold rolled $60 \%$ and annealed.

thetical texture of the rimmed steel which would produce the same level of deep drawability as the experimental sample (same average plastic strain ratio) but without a significant earing tendency. It is worth noting that an improvement effected by making $W_{440}$ zero by slightly increasing the proportion of $\{100\}\langle 011\rangle$ would lower the overall level of performance by reducing $r$. Some compromise would therefore seem to be inevitable in practice.

Variations in Plastic Anisotropy of Rimming Steel Produced by Manipulation of the Texture Coefficients

Figure 10 shows the predicted variation of the plastic strain ratio, $r$, with angle from the rolling direction using coefficients up to the fourteenth order, 

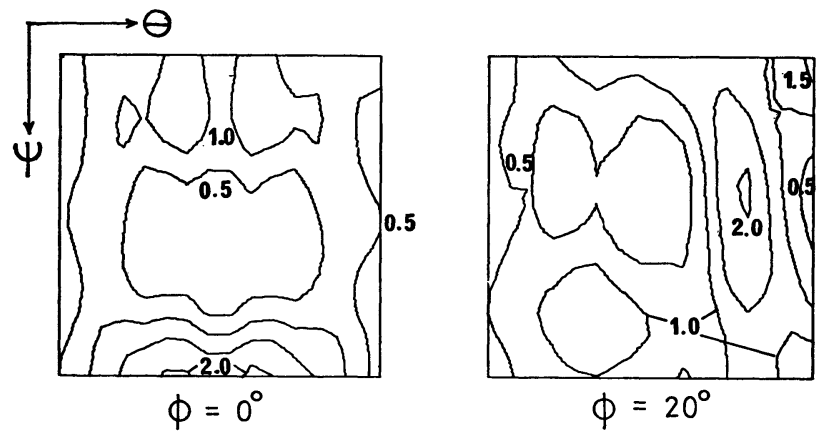

$\phi=20^{\circ}$

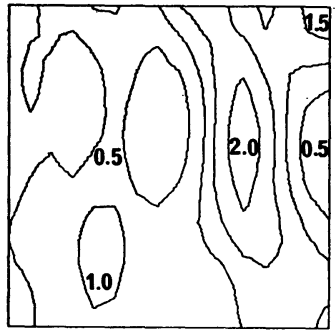

$\phi=25^{\circ}$

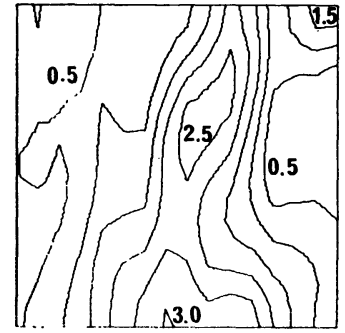

$\phi=45^{\circ}$

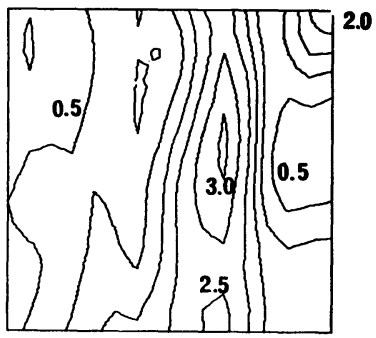

$\phi=35^{\circ}$

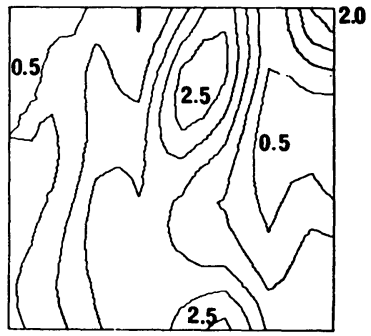

$\phi=55^{\circ}$

FIGURE 7 The crystallite orientation distribution function for rimmed steel cold rolled $60 \%$ and annealed with the $W_{420}$ coefficient set to zero.

for the various cases considered above. The unmodified curve exhibits considerable planar anisotropy, the $r$-value in the transverse direction being greater than that in the rolling direction. The results agree fairly well with the expected behaviour from similar steels. ${ }^{24}$ Removing $W_{420}$ means that $W_{440}$ alone controls the earing behaviour. This correlated well with the observed form of the predicted planar anisotropy. $W_{440}$ is seen to have the greatest influence on $r$. When both $W_{420}$ and $W_{440}$ are set to zero almost no planar anisotropy is predicted. This latter observation is further confirmation of the predominance of the fourth order coefficients in determining the plastic anisotropy.

The data of this and of the preceding section can readily be combined to define the developments to
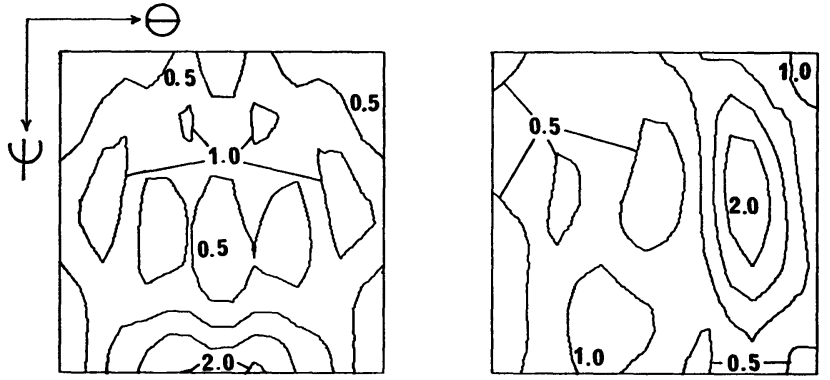

$\phi=0^{\circ}$

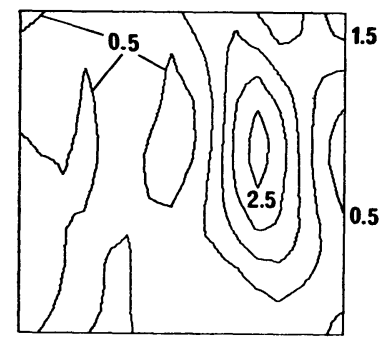

$\phi=20^{\circ}$

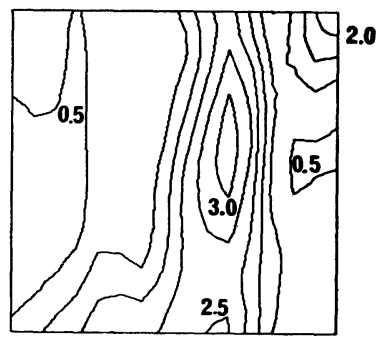

$\phi=25^{\circ}$

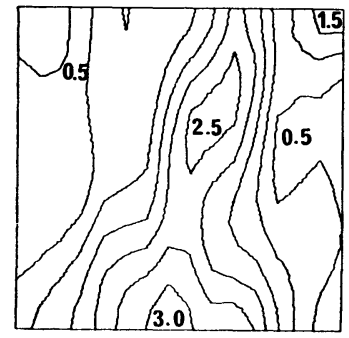

$\phi=45^{\circ}$

$\phi=35^{\circ}$

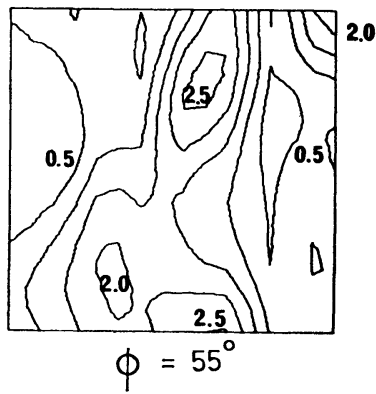

FIGURE 8 The crystallite orientation distribution function for rimmed steel cold rolled $60 \%$ and annealed with the $W_{440}$ coefficient set to zero.

be aimed for to produce steels of high drawability with low earing tendency.

\section{Applications to Killed Steel}

Figure 11 shows the variation in the $W_{400}, W_{420}$ and $W_{440}$ coefficients for the killed steel reduced by varying amounts and annealed. Comparison with Figure 1 shows that the overall variations are quite similar in rimmed and killed steels. The much larger $W_{400}$ values, however, reflect the superior drawability of killed steel. The variation of $W_{400}$ with cold reduction agreed well with the variation of average strain ratio obtained by $\mathrm{Held}^{25}$ for a killed steel given a series of reductions prior to annealing under similar rolling conditions, viz.: 

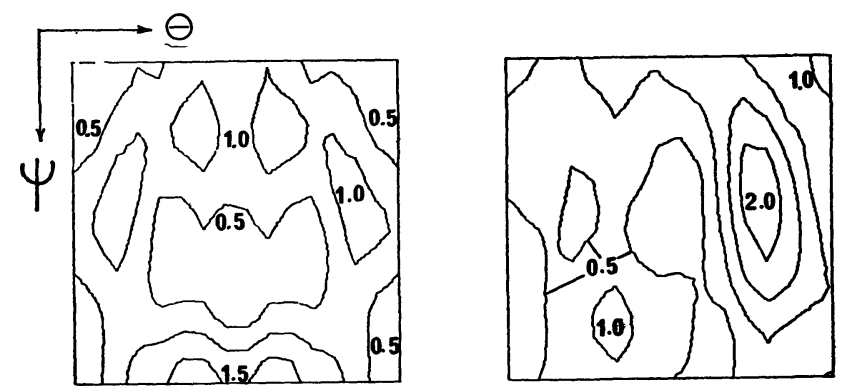

$\phi=0^{\circ}$

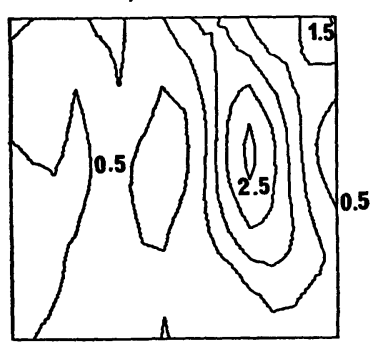

$\phi=25^{\circ}$

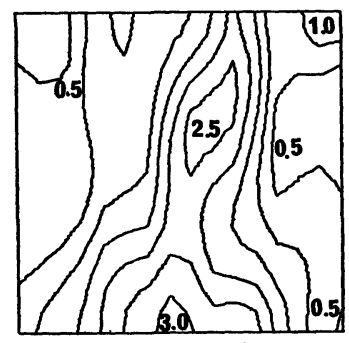

$$
\phi=45^{\circ}
$$

FIGURE 9 The crystallite orientation distribution function for rimmed steel cold rolled $60 \%$ and annealed with both the $W_{420}$ and $W_{440}$ coefficients set to zero.

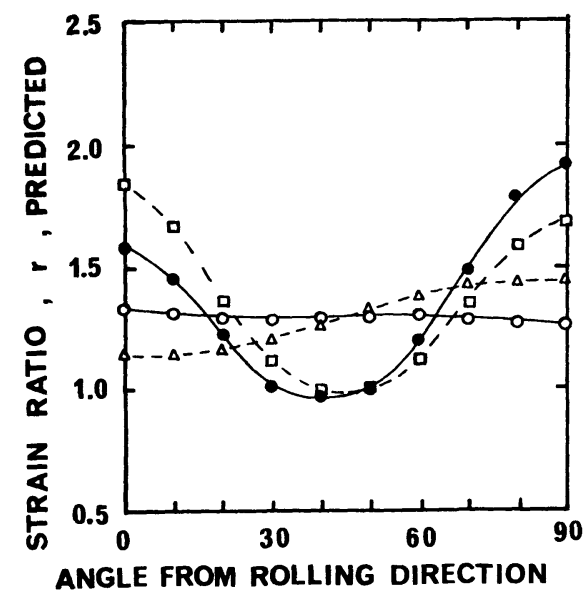

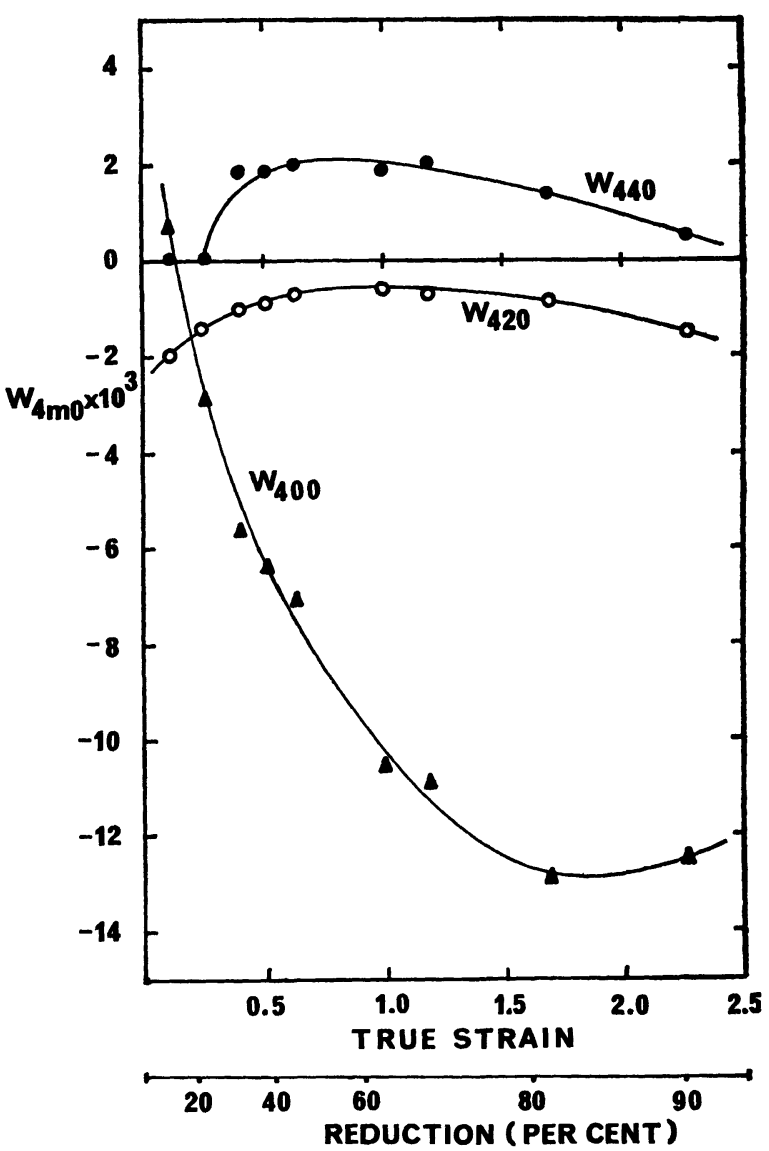

FIGURE 11 The variation of the $W_{4 m 0}$ texture coefficients with rolling reduction prior to annealing for the killed steel.

FIGURE 10 The predicted angular variation of the plastic strain ratio for the texture data of Figures 6, 7, 8, and 9.

predicted from experimental texture data.

$\square$ as with $W_{420}=0$.

$\triangle$ as with $W_{440}=0$.

$\bigcirc$ as with $W_{420}$ and $W_{440}=0$. 
good lubrication and heavy reductions on each pass.

Figure $12 \dagger$ shows the variation in ear height with rolling reduction for an aluminium stabilised steel similar to the one used to derive Figure 11. This is similar to Figure 2 for the rimmed steel. In general aluminium stabilised steel shows a greater degree of earing than rimming steel but this effect is not shown between the curves presented in Figures 2 and $12 .{ }^{24}$ Usually the lower critical cold reduction for zero earing is less for the killed steel than the rimmed, and the upper critical reduction higher.

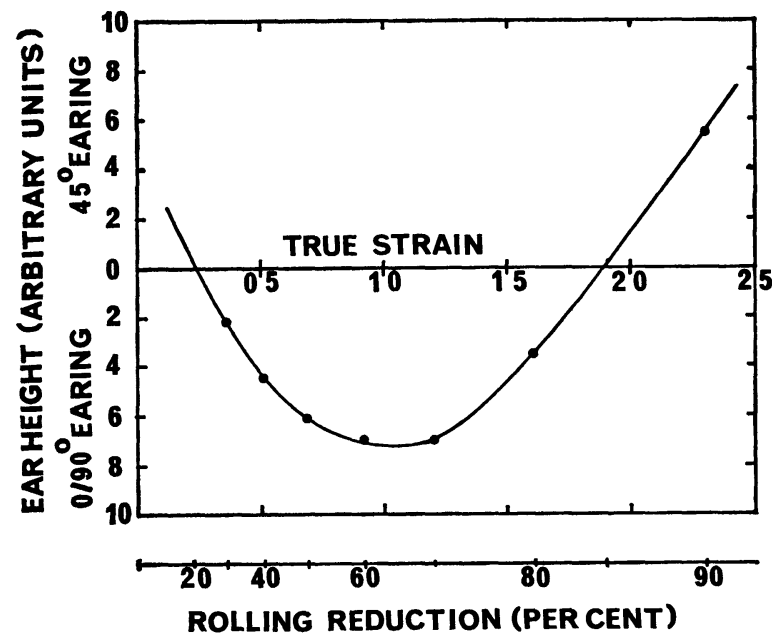

FIGURE 12 The variation in ear height with rolling reduction for an aluminium stabilised steel similar to the one used to derive Fig. 11.

Examination of the crystallite orientation distribution function for killed steel reduced by $40 \%$ and annealed (Figure 13) shows that the components described above which contribute to $W_{420}$ and $W_{440}$ are present. A specimen of killed steel cold rolled $40 \%$ prior to annealing was chosen since this treatment produced a similar overall level of texture severity as that of the rimmed steel cold rolled $60 \%$ and annealed (Figure 6). It can thus be deduced that the steps necessary to reduce the earing tendency in this steel are the same as those for the rimming steel.

\section{CONCLUSIONS}

1) $W_{400}$ is the single texture coefficient obtained from spherical harmonic analysis of texture data

†Data supplied by R. C. Hudd, British Steel Corporation, Strip Mills Division.
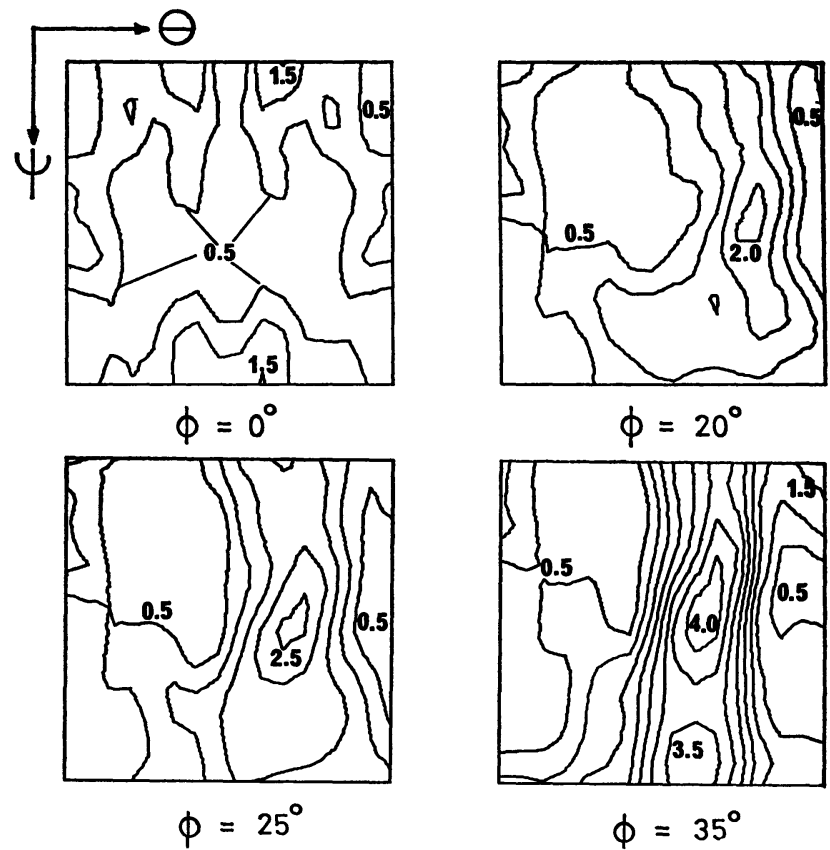

$\phi=35^{\circ}$
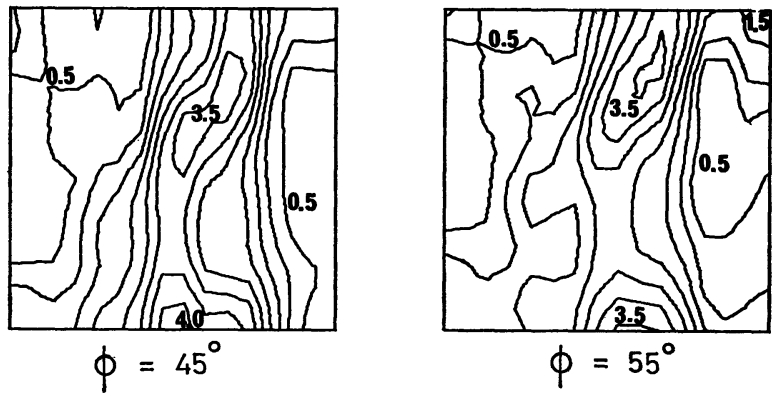

FIGURE 13 The crystallite orientation distribution function for killed steel cold-rolled $40 \%$ and annealed.

for commercial steels which most affects the overall normal anisotropy and drawing performance. $W_{420}$ and $W_{440}$ control the planar anisotropy and earing behaviour.

2) Components with $\{111\}$-planes in the plane of the sheet will produce the optimum normal anisotropy and associated maximum drawability. $\{110\}\langle u v w\rangle$ will have a deleterious effect.

3) The magnitudes of $W_{420}$ and $W_{440}$ are decided largely by the proportion of $\{h k l\}\langle 001\rangle$ components present.

4) To improve the earing behaviour some compromise with the overall drawability is necessary. Increasing the proportion of $\{100\}\langle 011\rangle$ will improve earing behaviour but reduce the drawability. However, reducing components near $\{110\}$ 
$\langle 001\rangle$ in steels should not significantly affect the drawability but is expected to reduce earing considerably.

\section{ACKNOWLEDGEMENTS}

The authors would like to thank the Science Research Council and the British Steel Corporation for financial support during the course of the research from which this paper evolved. They would also like to thank Mr. R. C. Hudd of the British Steel Corporation Strip Mills Division for supplying the steel and for stimulating discussions.

\section{REFERENCES}

1. R. L. Whiteley and D. E. Wise, in Flat Rolled Products III (Interscience, New York, 1962), pp. 47-63.

2. W. F. Hosford and W. A. Backofen, in Fundamentals of Deformation Processing (Syracuse University Press, New York, 1964), pp. 259-292.

3. D. V. Wilson, J. Inst. Metals 94, 84 (1966).

4. D. J. Blickwede, Trans. ASM 61, 653 (1968).

5. D. V. Wilson, Met. Reviews 14, 175 (1969).

6. J. C. Wright, Sheet Metal Ind. 42, 814 (1965).

7. J. Grewen, Proc. Polish Acad. Sci. (in press).
8. G. E. G. Tucker, Acta Met. 9, 275 (1961).

9. R. W. Vieth and R. L. Whiteley, see Refs. 5 and 7.

10. R. J. Roe, J. Appl. Phys. 36, 2024 (1965).

11. H. J. Bunge and W. T. Roberts, J. Appl. Cryst. 2, 116 (1969).

12. P. R. Morris and A. J. Heckler, in Advances in X-ray Analysis (Plenum Press, New York, 1968), Vol. 11, pp. 454-472.

13. H. J. Bunge, Krist. u. Technik 5, 145 (1970).

14. J. S. Kallend and G. J. Davies, J. Inst. Metals 99, 257 (1971).

15. H. Pursey and H. L. Cox, Phil. Mag. 45, 295 (1954).

16. G. J. Davies, D. J. Goodwill and J. S. Kallend, Met. Trans. 3, 1627 (1972).

17. J. F. W. Bishop and R. Hill, Phil. Mag. 42, 414 (1951).

18. J. F. W. Bishop and R. Hill, Phil. Mag. 42, 1298 (1951).

19. J. S. Kallend, Ph.D. Thesis, University of Cambridge (1970).

20. J. A. Elias and A. J. Heckler, Trans. Met. Soc. AIME 239, 1237 (1967).

21. G. J. Davies, D. J. Goodwill and J. S. Kallend, J. Appl. Cryst. 4, 67 (1971).

22. J. S. Kallend and G. J. Davies, J. Inst. Metals 98, 242 (1970).

23. R. S. Burns and R. H. Heyer, Sheet Metal Ind. 35, 261 (1958).

24. R. C. Hudd, Private Communication.

25. J. F. Held, Trans. Met. Soc. AIME 239, 573 (1967). 